\title{
Histopathological and immune-histochemical diagnosis of Burkitt Type Lymphoma in a cross bred cow
}

\author{
WLNV Vara Prasad ${ }^{1 *}$, K. Sujatha ${ }^{1}$, Ch Sri latha $^{1}$, D.Sreenivasulu ${ }^{2}$ and \\ K. Adilaxmamma ${ }^{3}$
}

${ }^{1}$ Department of Veterinary Pathology, ${ }^{2}$ Department of Veterinary Microbiology, ${ }^{3}$ Department of Veterinary Pharmacology, Sri Venkateswara Veterinary University, Tirupathi 517501.

*Corresponding author: PhD Scholar Email id: prasad.mudaliyar@ gmail.com; Mobile no: 9700221875.

Journal of Livestock Science (ISSN online 2277-6214) 10:81-85

Received on 26/6/2019; Accepted on 28/8/2019

doi. 10.33259/JLivestSci.2019.81-85

\begin{abstract}
Detailed pathomorphological study was carried out on tumor suspected mesenteric lymphnode from a Jersey cross bred cow. Uniform population of small non cleaved cells with prominent nucleoli were observed in cytological study, histopathology revealed characteristic starry sky appearence or moth eaten appearance of lymph node with diffuse infiltration of neoplastic cells in cortex and medulla. Immunohistochemistry shown positive reaction for common acute lymphocytic leukemia antigen (CD10), terminal deoxynucleotidyl transferase (TdT) and cluster of differentiation 5 (CD5) and negative reaction for cluster of differentiation 3 (CD3), B-cell lymphoma 2 (Bcl2), cluster of differentiation 20 (CD20) and cluster of differentiation 79a (CD79a). These Cytological, histopathological and immunohistochemical findings observed led to final diagnosis of burkitt lymphoma.
\end{abstract}

Key words: Tangible body macrophages; Burkitt type lymphoma; Common acute lymphocytic leukemic antigen. 


\section{Introduction}

Lymphomas are the most common spontaneous haematopoietic neoplasms in dogs and cats. They are used as animal model for human non-Hodgkin, lymphoma (Vezzali et al. 2009). Although lymphoid neoplasms were common in cattle next to epithelial neoplasms (Moulton, 1978) they were less studied. Lymphoid neoplasms in cattle may be spontaneous or may occur with bovine leukemia virus. Burkitt lymphoma is a high grade often extra nodal B cell lymphoma characterized by diffuse architecture. Recognition of burkitt lymphoma got significance in human oncology because first tumor shown to be curable and first shown associated with viral origin, Epstein barr virus (Gorczyca, 2014). The presence of lymphoma in cattle was first reported in India in the 1960s, till now no nation wise surveys have been reported. Although Vernau et al. 1992 reported presence of burkitt type lymphoma in cattle of Canada for the first time, detailed pathomorphological study on burkitt lymphoma in cattle was not recorded. In India, the occurrence of Burkitt type lymphoma in cattle has never been reported. The objectives of this study were to describe burkitt type of lymphoma in cross bred cattle and to conduct a pathomorphological and immunohistochemical study of this disease. The scoring of interphase Nucleolar orgainiser region (NOR) using the argyrophillic technique has been shown to have significant role in assessing the degree of malignancy in neopolasms (Smith et al. 1993). In the present study various specific immunohistochemical markers were utilized for identification and imunophenotypic charecterization. The panel of markers studied were based on morphological differential diagnosis which includes B Cell marker (CD20, Bcl2, CD 10 and CD79a), T cell marker (CD 3, CD5) and other markers TdT (marker of immature lymphoid cells).

\section{Materials and Methods}

A 6 years old Jersey crossbred cow necropsy was conducted at Department of Veterinary Pathology, College of Veterinary Science, Tirupathi, mesentric lymphnodes were suspected for lymphoma. Suspected lymphnode tissue samples were collected and preserved for further studies. Impression smears were collected from suspected lymphnode, fixed in methanol and they were stained with Giemsas (Drury et al. 1967). Tissue were fixed in $10 \%$ neutral buffered formalin and embedded in paraffin, and sections were cut at $5 \mu$ and stained with hematoxylin and eosin (HE) (Culling, 1974) and AgNOR (Krishnamurthi and Paliwal 1998). The expression of CD20, CD10, CD79a, Bcl2, TdT, CD3 and CD5 were estimated in Tissue using manual methods (Shi et al. 1991) with commercial available primary antibody (Biogenex India), Substrate and secondary antibodies (QD440-Xake, Bigenex India).

\section{Results and Discussion}

A 6 years old Jersey crossbred cow with a history of sudden death was presented for necropsy at Department of Veterinary Pathology, College of Veterinary Science, Tirupathi. Carcass was highly emaciated with visible bony prominence. Mesenteric lymph nodes are found to be greatly enlarged than its usual size. Grayish white areas without gross corticomedullary differentiation and surface swelling was seen in cut surface of neoplastic mesenteric Lymphnode (Fig. 1). Cytological imprint of suspected tissue shown uniform population of medium sized cells having small rounded non cleaved nuclei, granular chromatin with prominent nucleoli and thin rim of basophilic cytoplasm with small vacuoles (Fig. 2). Correspondingly Vernau et al. (1992) in cattle, Valli (2011) in dogs and Gorczyca (2014) in humans reported similar findings. Histopathological examination of lymphnode sections revealed moth eaten appearance with presence of more number of large histiocytes or tingible body macrophages with starry sky appearance (Fig. 3). Diffuse infiltration of uniform medium sized neoplastic lymphoid cells with small sized non cleaved nucleus were arranged in the form of sheets and bunches of grapes like pattern in cortical, paracortical and medullary region of lymphnode (Fig. 4). These were in accordance with Vezzali et al. (2009), Kelemen et al. (2010) and Kimura et al. (2011). Immunohistochemistry shown strong Immune reactivity with CD10 (Fig. 5), TdT co expression also observed (Fig. 6) and low immunoreactivity was observed with CD5 (Fig. 7). CD10 positive cells were diffusely present throughout lymphnode. Immuno reactivity with CD20, CD79a, CD3 and Bcl2 were found to be negative (Fig. 8, 9, 10 and 11). Vezzali et al. (2009), Kelemen et al. (2010) and Kimura et al. (2011) reported similar findings. Mitotic figures were infrequent. AgNOR staining revealed presence of nucleoli as black colored spots with in the nucleus of neoplastic cells. The mean AgNOR count obtained was 3 (Fig. 12).

Conclusion: Based on Cytological, histopathological, Immunohistochemical features and AgNOR staining, the present case was diagnosed as Burkitt type lymphoma. 


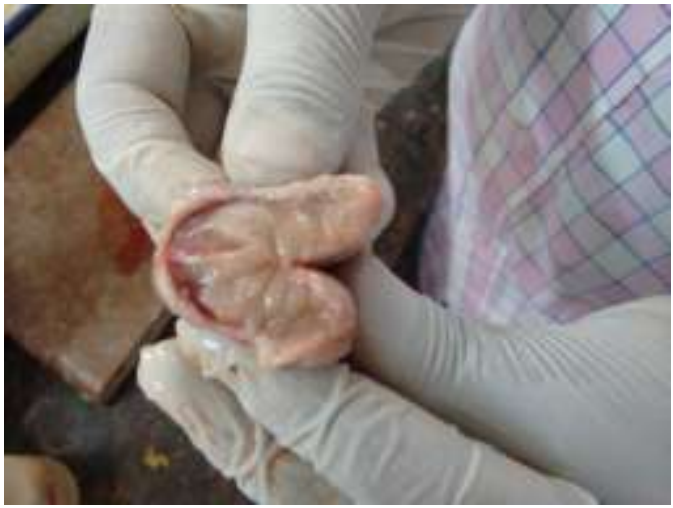

Fig. 1. A cut surface of neoplastic mesenteric lymphnode shows swelling over cut surface, grayish white in color and absence of cortico medullary demarcation.

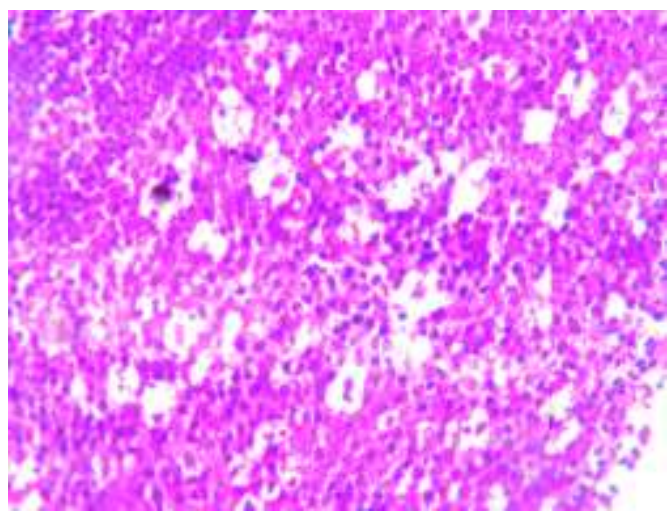

Fig.3. Neoplastic lymphnode cut surface shown presence of numerous tingible body macrophages and giving starry sky appearance. H \& E 400 X.

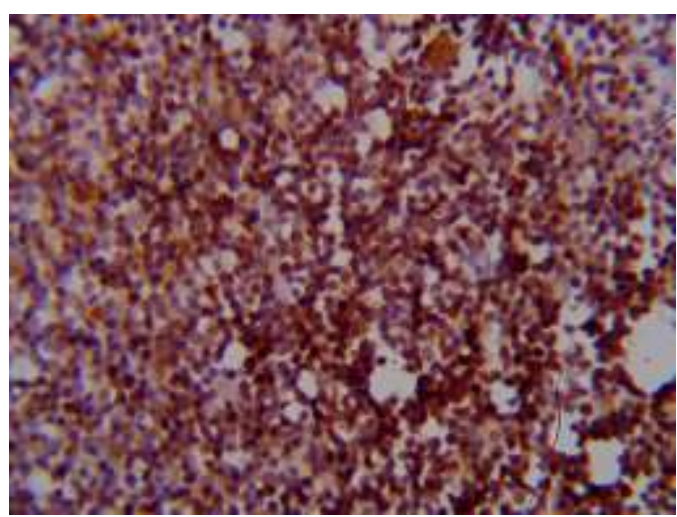

Fig. 5 Neoplastic mesenteric lymphnode shows strong immune reactivity with CD10 marker and Note diffuse distribution of neoplastic cells whose cytoplasm showing positive for CD 10 marker. Immunohistochemistry 400x

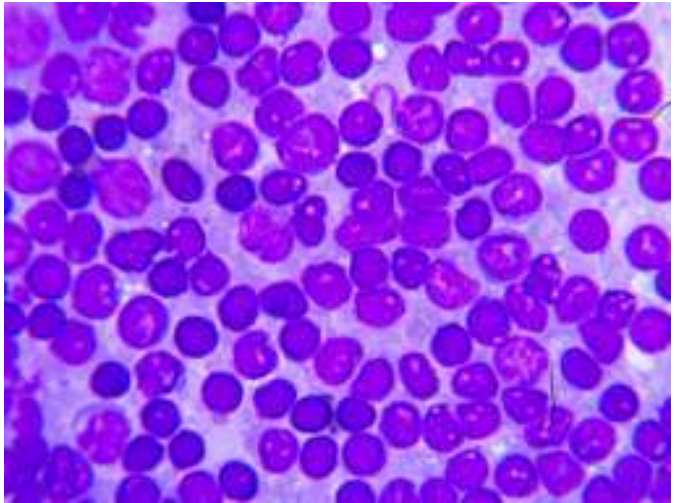

Fig. 2. Cytology of neoplastic mesenteric node reveals uniform population of medium sized cells with small round and non cleaved nucleus, nuclei are very transformed with multiple small and large nucleoli in a coarse chromatin material. Giemsas stain 1000x.

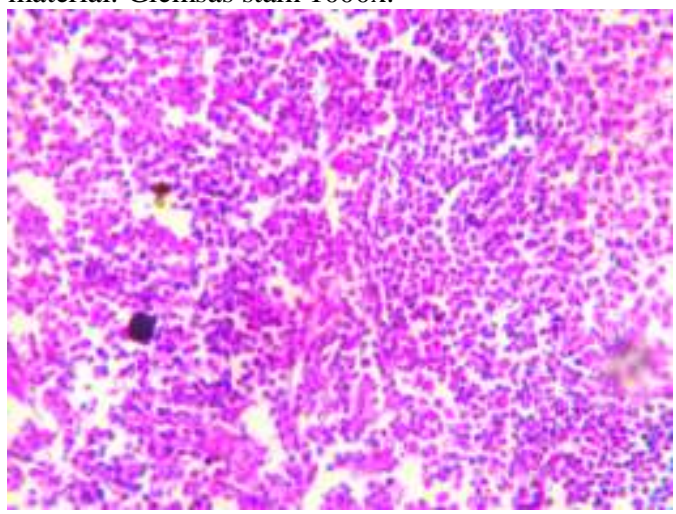

Fig. 4. Neoplastic lymphnode shows numerous uniform population of small non cleaved cells arranged in sheet like pattern (Right side) and aggregates of neoplastic cells arranged in bunches of grapes like pattern (Left side). H \& E $400 \mathrm{X}$.

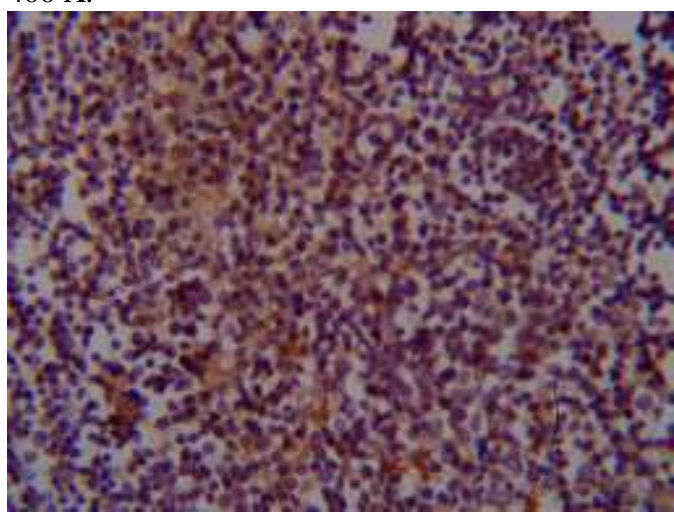

Fig. 6 Neoplastic mesenteric lymphnode shows low immune reactivity with CD5 marker. Immunohistochemistry 400x. 


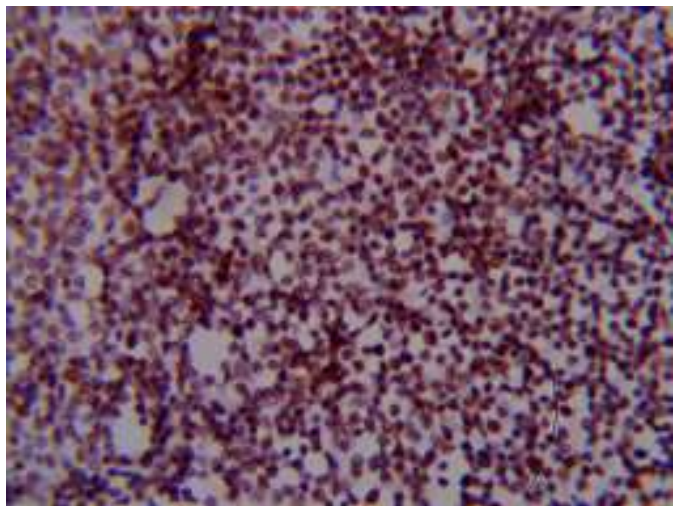

Fig. 7 Neoplastic mesenteric lymphnode shows strong immune reactivity with TdT marker and neoplastic cells were diffusely distributed and their nuclei are showing strong positive for TdT. Immunohistochemistry 400x.

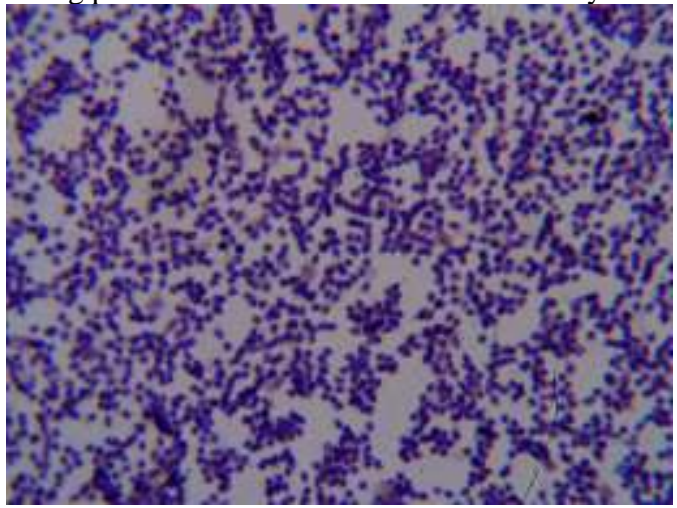

Fig. 9 Neoplastic mesenteric lymph node shows negative immune reactivity with $\mathrm{CD} 79 \mathrm{a}$ marker. Immunohistochemistry $400 \mathrm{x}$.

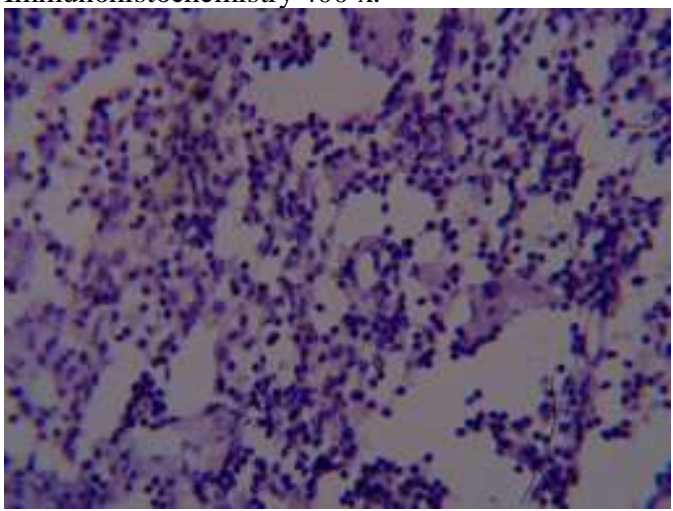

Fig. 11 Neoplastic mesenteric lymph node shows Negative immune reactivity with CD20 marker. Immunohistochemistry 400x.

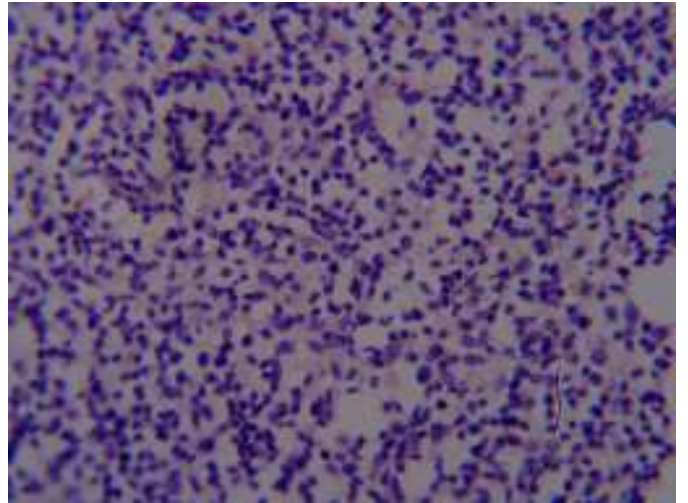

Fig. 8 Neoplastic mesenteric lymphnode shows negative immune reactivity with CD3 marker. Immunohistochemistry $400 x$.

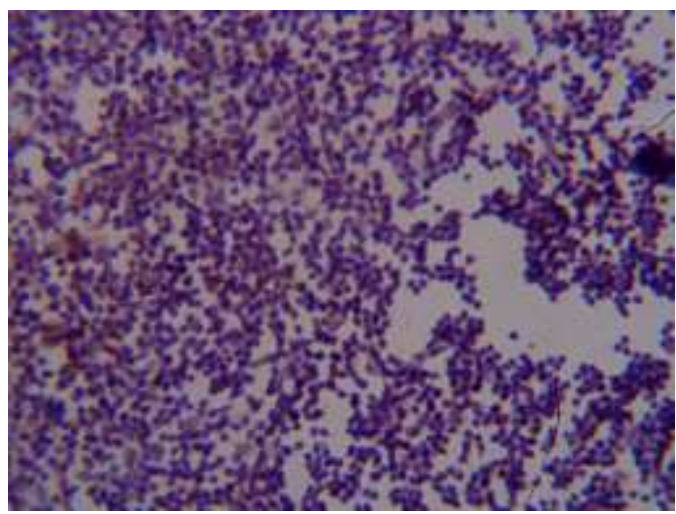

Fig. 10 Neoplastic mesenteric lymph node shows negative immune reactivity with $\mathrm{Bcl} 2$ marker. Immunohistochemistry $400 x$.

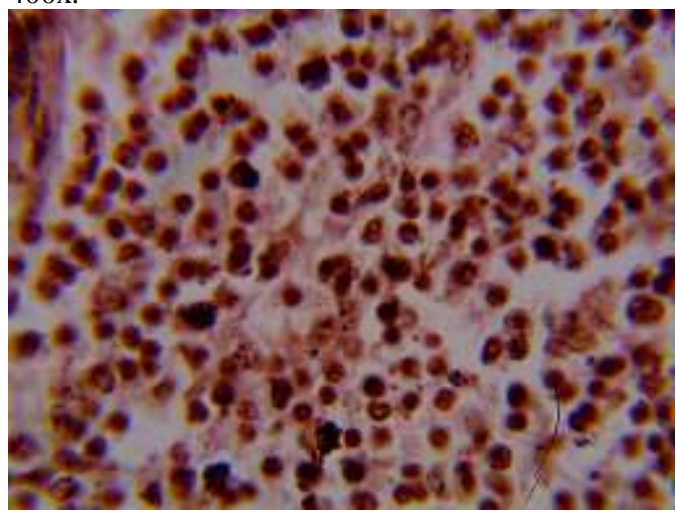

Fig 12 AgNOR staining revealed presence of small round dot like nucleoli. H\&E400x. 


\section{References}

1) Culling CFA 1974 Hand Book of Histopathological and Histochemical Techniques (Including Museum Techniques) $3^{\text {rd }}$ Edition. 36.

2) Drury RAB, Wallenton EA, Cameorn SR 1967. Carleton's Histological Technique. (4 ${ }^{\text {th }}$ edtn) Oxford University Press. New York / Toronto

3) Gorczyca W, 2014. Atlas of Differential Diagnostics in neoplastic hematology. CRC Press, Taylor \& Francis Group.

4) Kelemen K, Braziel RM, Gatter K, Bakke TC, Olson S, Fan G, 2010. Immunophenotypic variations of Burkitt Lymphoma. American Journal of Clinical Pathology 134: 127 - 138.

5) Kimura KC, Zanini DA, Nishiya AT, Dias RA, Dagli MLZ 2011. Morphology and immunophenotypes of canine lymphomas: a survey from the service of animal pathology, School of veterinary medicine and animal science, University of Sao Paulo, Brazil. Brazilian Journal of Veterinary Pathology 4(3): 199 206.

6) Krishnamurthi V, Paliwal OP 1998. Nuclear organizer region count as a diagnostic marker for tumors and cell proliferation rate in certain neoplasms of animals. Indian Journal of Veterinary Pathology 22(1): 610 .

7) Moulton JE 1978. Tumors in Domestic Animals. II Edn., University of California press, Berkeley, Los Angeles.

8) Shi SR, Key ME, Kalra KL 1991. Antigen retrieval in formalin-fixed, paraffin-embedded tissues: an enhancement method for immunohistochemical staining based on microwave oven heating of tissue sections. Journal of Histochemistry and Cytochemistry. 39(6): - $741-748$.

9) Smith FG, Murray PG, Crocker J 1993. Correlation between PCNA and AgNOR scores in non-Hodgkin's lymphomas using sequential staining technique. Journal of Clinical Pathology 46(1): 28 - 31 .

10) Valli VE, Myint MS, Barthel A, Bienzle D, Caswell J, Colbatzky F, Durham A, Ehhart EJ, Johnson Y, Jones C, Kiupel M, Labelle P, Lester S, Miller M, Moore P, Moroff S, Roccabianca P, Vara JR, Ross A, Scase T, Tvedten H,Vernasu W 2011. Classification of canine malignant Lymphomas according to World Health Organisation criteria. Veterinary Pathology 48(1): $198-211$.

11) Vernau W, Jacobs M, Valli VEO, Iieeney JL 1997 Brief Communications and Case Reports The Immunophenotypic characterization of bovine lymphomas. Veterinary Pathology 34: 222 - 225.

12) Vezzali E, Parodi AL, Marcato PS, Bettini G. 2009. Histopathologic classification of 171 cases of canine and feline non Hodgkin lymphoma according to the WHO. Veterinary and Comparitive Oncology 8(1): $38-49$. 